\title{
Preoperative markers in differentiated thyroid cancer
}

\section{Alexandra Chrisoulidou}

\section{Department of Endocrinology, Theagenio Cancer Hospital, Thessaloniki, Greece}

\section{Dear Editor,}

I read with great interest the article by Yazici et $\mathrm{al}^{1}$ on $\mathrm{TSH} / \mathrm{Tg}$ ratio in the prediction of thyroid cancer. The justification for thyroid surgery in patients with thyroid nodules has led to many interesting studies looking into predictive markers, albeit without as yet concrete results that could alter the current practice.

In the study by Yazici et al, 68 patients had thyroid cancer confirmed by histopathology. Thirty-two of these patients harbored papillary microcarcinomas (PMCs), which account for nearly $50 \%$ of all cancers studied. The authors pointed out in the discussion section that a limitation of their study might be that tumor diameter was not recorded. I strongly agree that tumor size is critical in such studies. PMCs represent a distinct group of thyroid tumors that are elusive to preoperative assessment. ${ }^{2}$ This subset of tumors differs so much from larger thyroid cancers in terms of

\section{Address for correspondence:}

Alexandra Chrisoulidou, MD, PhD, Department of

Endocrinology, Theagenio Hospital, 2 Al. Simeonidi street,

54007 Thessaloniki, Greece; e-mail: alexri@otenet.gr

Received: 25-09-2016, Accepted: 03-10-2016 recurrence rates ${ }^{3}$ and survival that treatment policies are currently changing towards more conservative approaches. ${ }^{4}$ Therefore, a heterogeneous study group including from minute intrathyroidal tumors to invasive large cancers may not help us find true preoperative markers. We need to pose specific questions in order to get validated results.

\section{REFERENCES}

1. Yazici P, Mihmanli M, Bozkurt E, Ozturk FY, Uludag M, 2016 Which is the best predictor of thyroid cancer: thyrotropin, thyroglobulin or their ratio? Hormones (Athens) 15: 256-263.

2. Leboulleux S, Tuttle RM, Pacini F, Schlumberger M, 2016 Papillary thyroid microcarcinoma: time to shift from surgery to active surveillance? Lancet Diabetes Endocrinol 4: 933-942.

3. Ghossein R, Ganly I, Biagini A, Robenshtok K, Rivera M, Tuttle RM, 2014 Prognostic factors in papillary microcarcinomas with emphasis on histologic subtyping: a clinicopathologic study of 148 cases. Thyroid 24: $245-253$.

4. Haser GC, Tuttle RM, Su HK, et al, 2016 Active surveillance for papillary microcarcinoma: new challenges and opportunities for the health care system. Endocr Pract 22: 602-611.

\section{Dear Editor}

We are grateful to A. Chrisoulidou for pointing out the importance of including tumor size as an auspicious predictive marker of thyroid cancer. We presented this issue in the manuscript as a limitation of our study. Currently, apart from fine needle aspiration biopsy, there is increasing interest in the development of novel easy-to-apply markers for enhancing the likelihood of early detection of thyroid malignancy. Nevertheless, most of the new advanced methodologies, e.g. genetic markers, are costly to perform and require technical expertise for evaluation of findings. 
In recent years, among several preoperative biochemical markers such as thyroglobulin (Tg), anti-Tg antibody, free T4 and TSH level, particularly Tg and TSH have been proposed as predictors of thyroid cancer. ${ }^{1}$ While tumor size was not an independent predictor of thyroid cancer, in particular for nodules $>1.7 \mathrm{~cm}$ in size, elevated serum $\mathrm{Tg}$ predicted the risk of malignancy (Lee et al). Petric et al pointed out the relationship between tumor size and thyroglobulin level; however, their study included all tumors $<2$ $\mathrm{cm}$ in size. ${ }^{2}$

Few studies have investigated the role of the ratios of these biomarkers in predicting thyroid malignancy. Interestingly, these_studies did not take into account tumor size as a predictive marker in particular. Wang et al found that increasing preoperative serum $\mathrm{TSH}: \mathrm{Tg}$ ratio was a risk factor for thyroid carcinoma. ${ }^{3}$ In their study, nodule size was not investigated in particular, although the average size was found to be $17 \mathrm{~mm}$ in the patients with thyroid malignancy. On the other hand, Cohen et al, in evaluating the role of Tg:TSH ratio in the prediction of malignancy, showed that this ratio was eligible only for a papillary thyroid tumor measuring $10 \mathrm{~mm}$ or larger. ${ }^{4}$

In our series, as stated in your letter, almost half of the papillary tumors were micropapillary cancer. This sample size was relatively small, especially for a stratified analysis; therefore, we could not take into consideration the nodule size and characteristics for statistical analysis. Nevertheless, our results revealed that TSH:Tg was likely to play an important role in predicting thyroid malignancy.

In conclusion, we would like to state that the published study is ongoing and more detailed information regarding nodule characteristics and patient groups are being documented. Taking into account the nodule size as a predictive marker may be helpful for identifying those patients who are at a higher risk for thyroid cancer.

\section{Sincerely yours, Pinar Yazici, MD}

\section{REFERENCES}

1. Lee EK, Chung KW, Min HS, et al, 2012 Preoperative serum thyroglobulin as a useful predictive marker to differentiate follicular thyroid cancer from benign nodules in indeterminate nodules. Korean Med Sci 27: 1014-1018.

2. Petric R, Perhavec A, Gazic B, Besic N, 2012 Preoperative serum thyroglobulin concentration is an independent predictive factor of malignancy in follicular neoplasms of the thyroid gland. J Surg Oncol 105: 351-356.

3. Wang L, Li H, Yang Z, Guo Z, Zhang Q, 2015 Preoperative serum thyrotropin to thyroglobulin ratio is effective for thyroid nodule evaluation in euthyroid patients. Otolaryngol Head Neck Surg 153: 15-19.

4. Cohen N, Pakdaman MN, Rochon L, Tamilia M, Payne JR, 2009 Tg:TSH Ratio as a Tumor Marker for Thyroid Cancer. Otolaryngol Head Neck Surg 141: 165. 